\title{
The influence of the morphometric parameters of the intercondylar notch on occurrence of meniscofemoral ligaments
}

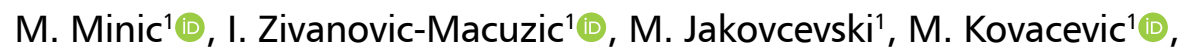 \\ S. Minic ${ }^{2}$, D. Jeremic ${ }^{1}$ (1) \\ ${ }^{1}$ Department of Anatomy, Faculty of Medical Sciences, University of Kragujevac, Serbia \\ ${ }^{2}$ College of Applied Health Sciences, Ćuprija, Serbia
}

[Received: 7 July 2020; Accepted: 9 November 2020; Early publication date: 30 December 2020]

\begin{abstract}
Background: The purpose of this study was to examine the existence of correlation between the morphometric parameters of the intercondylar notch of the femur and the occurrence of meniscofemoral ligaments (MFLs) and if there is any relationship in the running angle $(R A)$ value between narrowed and normal sized intercondylar notch.
\end{abstract}

Materials and methods: Coronal, sagittal and horizontal magnetic resonance (MR) images of 90 patients with specified exclusion criteria were included in this study. The $\chi^{2}$ test was used for statistical analysis. In our research either one or both MFLs were identified in $70(77.8 \%)$ of the 90 coronal MR images. In normal sized intercondylar notch, MFLs was seen in 39 (43.3\%) cases and on 31 (34.4\%) MR images with narrowed intercondylar notch.

Results: A significant correlation was established between the occurrence of the MFL and morphometric parameters of the intercondylar notch $(p<0.05)$. In normal sized intercondylar notch, 12 posterior meniscofemoral ligaments ( $P M F L s$ ) of type I were detected (RA value $42^{\circ}$ ), 8 of type II (RA value $\left.33^{\circ}\right)$, 5 of type III (RA value $23^{\circ}$ ) and two were of indeterminate type, whilst 10 anterior meniscofemoral ligaments (aMFLs) were of type I (RA value $\left.39^{\circ}\right)$, 7 of type II (RA value $31^{\circ}$ ), 2 of type III (RA value $25^{\circ}$ ) and the remaining 6 were indeterminate. In narrowed intercondylar notch, 10 ligaments of pMFLs were of type I (RA value $30^{\circ}$ ), 8 of type II (RA value $\left.25^{\circ}\right), 5$ of type III (RA value $20^{\circ}$ ), 10 ligaments of aMFLs were of type I (RA value $3^{\circ}$ ) and 9 were indeterminate. Statistically significant differences in the value of the running angle of PMFL type I and of type II were evaluated between two groups with different shaped intercondylar notch $(p<0.05)$.

Conclusions: The results shown in our study may be useful in medical clinical practice, reconstructive surgery, interpretation of knee $M R$ images as well as genetic research. (Folia Morphol 2022; 81, 1: 190-195)

Key words: intercondylar notch, meniscofemoral ligament, knee and magnetic resonance 


\section{INTRODUCTION}

The intercondylar notch (IN) is located on the posterior side of the distal part of the femur between the lateral and medial condyle. The roof of the notch is built by the distal extremity of the femur while its distal and posterior borders are marked by distal surface of condyles [20]. Anderson et al. [4] found that the females have a smaller IN dimensions compared to males but they found no difference in shape of the notch. Based on morphometric parameters of IN, Hutchinson et al. [14] defined two types of IN, U-shaped and A-shaped notch, while Tanzer and Lenczner [21] considered narrowed IN, as A-shaped and IN with normal with as U-shaped notch.

The variation in morphology of the femur is associated with increased risk for ligament injury. Of the ligaments in the knee joint, the anterior cruciate ligament $(A C L)$ is the most commonly injured $[19,20$, 25]. Intercondylar notch dimensions are considered as a significant predictive risk factor for ACL injury [5]. The meniscofemoral ligaments (MFLs) connect the posterior horn of the lateral meniscus to the intercondylar aspect of the medial femoral condyle, or to the posterior cruciate ligament [23]. There are two types of MFLs, depending on their position in relation to the posterior cruciate ligament (PCL). According to Amis et al. [1] the ligament of Humphry (anterior meniscofemoral ligament [aMFL]) passes anterior to the PCL and attaches distally, close to the articular cartilage. The ligament of Wrisberg (posterior meniscofemoral ligament [pMFL]) passes posterior to the $\mathrm{PCL}$ and attaches proximally, close to the roof of the IN [1, 2]. The aMFL is tense in knee flexion, while the PMFL is tense in knee extension. [9]. One MFL is present in $93 \%$ of specimens, whilst both ligaments co-exist more frequently in younger specimens $[10,11,24]$. According to earlier anatomical studies their incidence may range from $35 \%$ to $76 \%$ [12]. The attachments of those two ligaments are separated, which supports the theory that those are two separate structures and not, as was previously thought, two branches of the same ligament [15]. The other study suggests that they may degenerate with age [18]. It has previously been demonstrated that additional resection of the MFL further destabilised the knee joint, as well as significantly increased anterior tibial translation $[8,17]$.

In literature data, depending on the location of the attachment, there are three types of MFLs: 1) the ligament of type I, inserted into the medial femoral condyle and separated from the PCL; 2 ) the ligament of type II, attached to the proximal portion of the $\mathrm{PCL} ; 3)$ the ligament of type III, attached to the distal portion of the PCL [7]. Cho et al. [7] defined the running angle of the MFL as the angle between the line connecting the distal surfaces of medial and lateral femoral condyles and the long axis of the MFL. The relationship between morphometric parameters of the IN and the running angle value was reported [7]. However, the influence of the morphometric parameters of the IN on the value of the running angle is poorly described in literature. On the other hand, this information could be relevant in predicting the risk of MFLs rupture and their degeneration.

The aim of this study was to determinate occurrence of MFLs depending on the IN morphometry, to examine incidence of single and both MFLs as well the absence of MFLs, to evaluate statistically significant difference between the different type of IN and finally to determinate influence of its morphology on the value of the running angle.

\section{MATERIALS AND METHODS}

The study included magnetic resonance (MR) images of 90 patients ( 48 males and 42 females), aged from 20 to 60 (44.68 \pm 10.52$)$ receiving a 1.5-T knee scan at our radiologic institute after the approval from ethics committee, which were taken in the period from 2010 to 2017. All MR examinations were performed on 1.5-T MR unit (Siemens Area AG, model syngo MR E11, with NUMARIS/4 software, Siemens software packages). The knee was placed in neutral position in an extremity coil. Coronal images were obtained using conventional spin-echo techniques (time to echo: $8.7 \mathrm{~ms}$, repetition time: $3080 \mathrm{~ms}$, field of view: $190 \times 190 \mathrm{~mm}$, section thickness: $3.5 \mathrm{~mm}$, with $1 \mathrm{~mm}$ gap, voxel size: $0.6 \times 0.6 \times 3.5 \mathrm{~mm}$ ). Additionally, for the measurements, we used Image J $1.50 \mathrm{~g}$ software (National Institutes of Health, USA). The following exclusion criteria were defined:

— inadequate quality of the images;

- fracture or dysplasia of the distal femur;

- previous arthroscopy or open surgery;

- osteoarthritic changes of the IN;

- varus or valgus deformity of the knee.

The following parameters on horizontal images (Fig. 1) were measured: the width of the lateral and medial femoral condyle, the notch width (NW), the total width of the distal femur. The cross section (a) on which measurements were conducted was determined on sagittal MR images, based on the vertical 


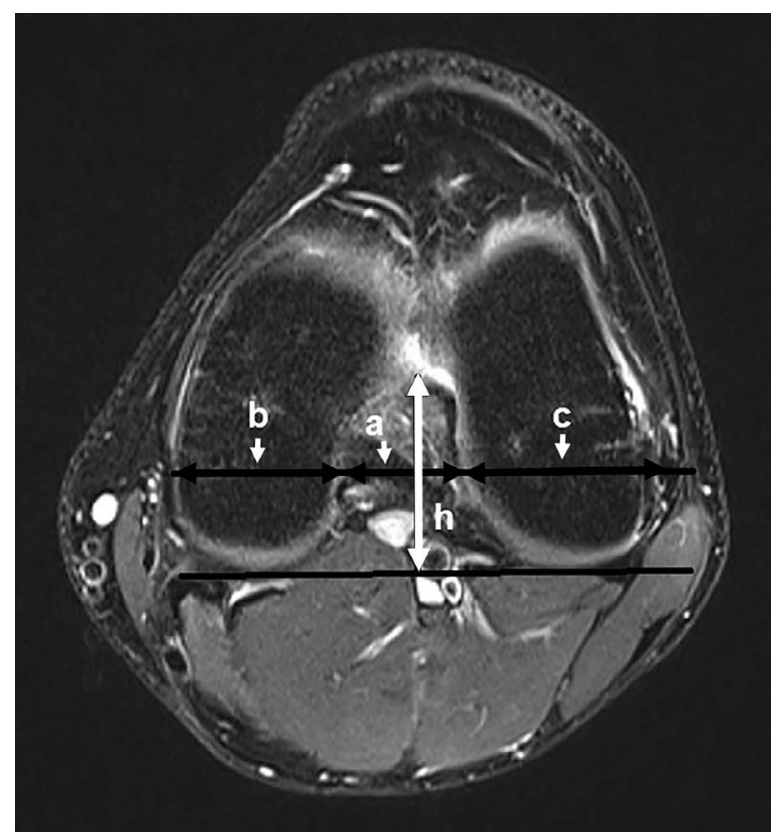

Figure 1. Intercondylar notch - A-shaped. The notch width (a), width of the medial femoral condyle (b), width of the lateral femoral condyle (c) and the notch height $(\mathrm{h}$ - white line with arrow on both ends) on axial magnetic resonance image in a 30-year-old man.

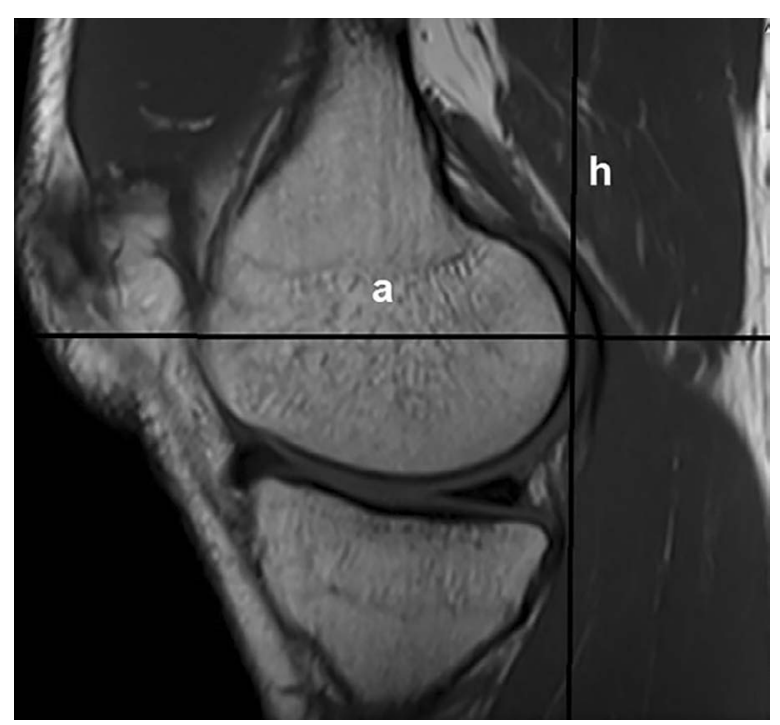

Figure 2. Cross section (a) passes through the most posterior point of the lateral and medial condyle ( $\mathrm{h}$ - vertical line) on sagittal magnetic resonance image.

line (h) which passes through the most posterior point of the lateral and medial condyle (Fig. 2). At this level, on axial images, the measurements were done on the line which passes through the popliteal groove. The width of the medial femoral condyle (b) and the width of the lateral femoral condyle (c) were measured as the distance between the external and internal margins of each condyle and the NW was measured as the distance between the inner most margins of femoral condyles (a). Also, the notch height $(\mathrm{ICH})$ was measured on the line perpendicular to the line constructed through the most posterior point of the lateral and medial condyle of the femur, as a distance between the apex of the IN and the intersection of above mentioned lines (h) (Fig. 1).

The notch width index (NWI) - the ratio between the NW and the total width of the distal femur, was calculated. The values of the NWI of 0.270 or more were considered as normal, while values of 0.269 or less were considered as below normal [3]. Notch shape index (NSI) was defined as the ratio between the NW and the ICH. The values of the NSI of 0.532 or less indicate on stenosed type of the IN [22]. We measured the running angle of the MFL as the angle between the line connecting the distal surfaces of medial and lateral femoral condyles and the long axis of the MFL on coronal images, using in literature described method (Fig. 3) [7]. Authors have noted no significant difference in the value of the running angle between the ligament of Humphery and the ligament of Wrisberg [7], so we used these criteria for both ligaments.

All measurements were done by two independent investigators. No significant difference was found in comparison of their measurements. In order to avoid bias, the measurements were repeated by both examiners after 3 weeks on the same, randomly selected $50 \mathrm{MR}$ images. The results were compared by Cohen kappa coefficient, with kappa values over the 0.8 for all measurement, which suggest very good intra- and interobserver agreement.

\section{Statistical analysis}

The statistical differences between the presence and absence MFLs as well as prevalence of A- or U-shaped IN on MR images were evaluated by $\chi^{2}$ tests. A p value of less than 0.05 was regarded as statistically significant.

For every metric variable the mean, standard deviation and range were displayed. The normal distribution was tested with the Kolmogorov-Smirnov test, as well as visually through a Gaussian distribution curve over the histogram of the respective data. The homogeneity of variances was ensured through a non-significant Levene's test. For normally distributed, metric variables, differences between the groups were tested for significance with Student's t-test. In 


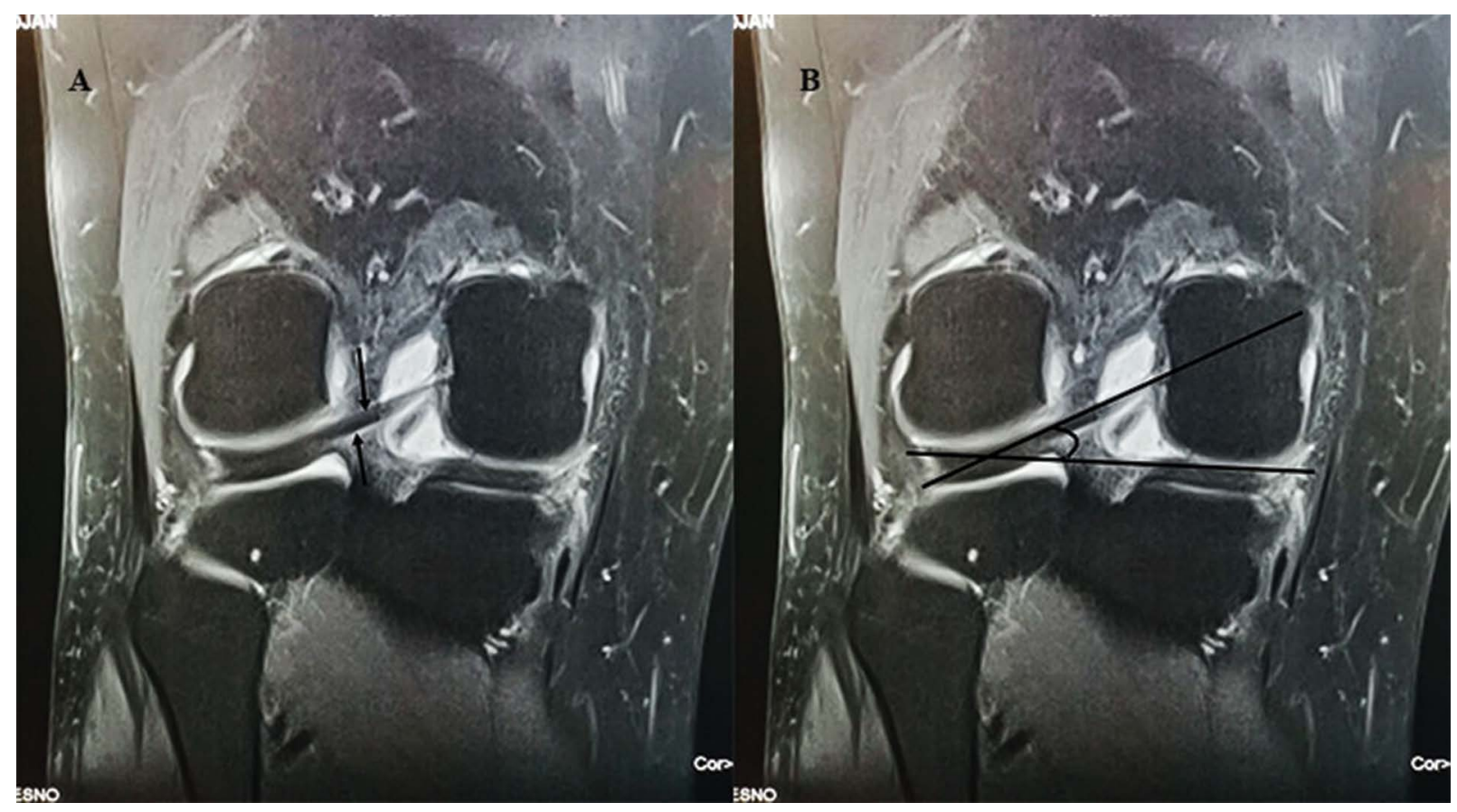

Figure 3. A. Coronal magnetic resonance image shows the meniscofemoral ligament (arrow); B. The running angle of the meniscofemoral ligament as the angle between the distal surfaces of medial and lateral condyles and long axis of the meniscofemoral ligament on coronal magnetic resonance images.

the case of non-normally distributed, metric variables, the Mann-Whitney U-test was used instead.

\section{RESULTS}

According to NWI and NSI criteria we found U-shaped IN in 46 (51.2\%) analysed coronal MR images and A-shaped IN in 44 (48.8\%) (Table 1). There was no significant difference in incidence between the groups $(p>0.05)$. The MFLs was identified in $70(77.8 \%)$ of the 90 coronal MR images scans. In group with U-shaped IN, MFLs was observed in $39(43.3 \%)$ cases and in 31 (34.4\%) cases in the group with A-shaped IN. There was a significant difference in MFLs occurrence between the groups with A- and U-shaped IN ( $p<0.05)$.

U-shaped. The pMFL was seen alone in $30.4 \%$ (14) of cases, while aMFL was observed alone in $26.1 \%$ (12). Both ligaments were visible in $28.3 \%$ (13) while neither was found in $15.2 \%$ (7) cases. Among the pMFL, 12 ligaments were of type I (average running angle [RA] value $42^{\circ}$ ), 8 of type II (average RA value $33^{\circ}$ ), 5 of type III (average RA value $23^{\circ}$ ) and 2 were classified as indeterminate type, whilst 10 ligaments of aMFL were of type I (average RA value $39^{\circ}$ ), 7 of type II (average RA value $31^{\circ}$ ), 2 of type III (average RA value $25^{\circ}$ ) and the remaining 6 were indeterminate. Statistically significant difference in the value of the RA was observed between the types
Table 1. Mean magnetic resonance images measurements of the distal femur

\begin{tabular}{lcc}
\hline & \multicolumn{2}{c}{ Intercondylar notch } \\
\cline { 2 - 3 } & $\begin{array}{c}\text { U-shaped } \\
\text { (n= 46) }\end{array}$ & $\begin{array}{c}\text { A-shaped } \\
\text { (n = 44) }\end{array}$ \\
\hline Intercondylar notch width $[\mathrm{mm}]$ & $21.23 \pm 2.28$ & $19.98 \pm 1.75$ \\
Intercondylar notch height $[\mathrm{mm}]$ & $36.77 \pm 1.67$ & $37.95 \pm 1.43$ \\
Total width of distal femur $[\mathrm{mm}]$ & $76.27 \pm 7.10$ & $76.45 \pm 6.23$ \\
Notch shape index & $0.577 \pm 0.021$ & $0.526 \pm 0.018$ \\
Notch width index & $0.278 \pm 0.023$ & $0.261 \pm 0.012$ \\
\hline
\end{tabular}

$(p<0.05)$. No significant differences in the value of the RA between the PMFL and aMFL were noted ( $p>0.05$ ).

A-shaped. In A-shaped IN single pMFL was detected in $27.3 \%$ (12) of cases and the single aMFL ligament was observed in $18.2 \%$ (8). Both ligaments were found in $25 \%$ (11) while neither was found in $29.5 \%$ (13). Statistically significant difference in the MFLs not detected group was observed between the $A$ and $U$ form of IN $(p<0.05)$. Among the pMFLs, 10 ligaments were of type I (average RA value $30^{\circ}$ ), 8 of type II (average RA value $25^{\circ}$ ), 5 of type III (average RA value $20^{\circ}$ ), while 10 ligaments of aMFLs were of type I (average RA value $35^{\circ}$ ) and 9 were indeterminate. Statistically significant differences in the value of the running angle were noted between the type I and type II $(p<0.05)$. 
Table 2. Incidence of the meniscofemoral ligaments (MFLs) and the values of its running angle (RA) in U-shaped and A-shaped intercondylar notch (IN)

\begin{tabular}{|c|c|c|c|c|c|c|c|}
\hline \multirow[t]{2}{*}{ Ligament (n) } & \multirow[t]{2}{*}{$U(n)$} & \multirow[t]{2}{*}{$A(n)$} & \multicolumn{2}{|c|}{ U-shaped IN } & \multicolumn{2}{|c|}{ A-shaped IN } & \multirow[t]{2}{*}{$\mathbf{P}$} \\
\hline & & & MFL type (n) & RA value $\left({ }^{\circ}\right)$ & MFL type (n) & RA value $\left({ }^{\circ}\right)$ & \\
\hline \multirow[t]{4}{*}{ Posterior MFL (50) } & 27 & 23 & Type I (12) & $42 \pm 3.9$ & Type I (10) & $30^{\circ} \pm 2.6$ & $p<0.05^{*}$ \\
\hline & & & Type II (8) & $33 \pm 2.3$ & Type II (8) & $25^{\circ} \pm 2.2$ & $p<0.05^{*}$ \\
\hline & & & Type III (5) & $23 \pm 2.5$ & Type III (5) & $20^{\circ} \pm 1.6$ & $p>0.05$ \\
\hline & & & ID type (2) & - & ID type (/) & - & \\
\hline \multirow[t]{4}{*}{ Anterior MFL (44) } & 25 & 19 & Type I (10) & $39 \pm 2.7$ & Type I (10) & $35^{\circ} \pm 2.5$ & $p>0.05$ \\
\hline & & & Type II (7) & $31 \pm 2.5$ & Type II (/) & - & \\
\hline & & & Type III (2) & $25 \pm 1.2$ & Type III (/) & - & \\
\hline & & & ID type (6) & - & ID type (9) & - & \\
\hline
\end{tabular}

${ }^{*}$ Statistically significant difference; U — normal sized intercondylar notch; A — narrowed intercondylar notch; ID — indeterminate type of MFL

Statistically significant differences in the value of the running angle of pMFL type I, between $\mathrm{U}$ - and A-shaped IN was observed ( $p<0.05$, Table 2$)$.

\section{DISCUSSION}

Earlier studies of the MFLs pointed different incidence of the aMFL and PMFL. The differences can be also found between the MR images and cadaveric studies. Herzog et al. [13] found that the MR images measurements were the closest to directly measured in the cadaver. Gupte et al. [10] conducted MR images study in which $93 \%$ specimens contained at least one MFL. The aMFL was present in $74 \%$, and the PMFL in $69 \%$. Both ligaments were present in $50 \%$. Bintoudi et al. [6] found the MFLs in $37 \%$ of cases, while in $7.6 \%$ of cases MFLs were absent. In MR images study performed by Cho et al. [7] the PMFL was identified in $90 \%$ of cases and aMFL was observed in $17 \%$. Both ligaments were visible in $15 \%$ cases and neither was seen in $7.5 \%$. Kusayama et al. [16] showed the MFL with incidences of $100 \%$ and that $46 \%$ of the specimens had both MFLs, $23 \%$ had only aMFL and the remaining $31 \%$ had a pMFL. Röhrich et al. [18] reported that the MFL exists in incidences of $94 \%$, aMFL in incidences of $71 \%$ as was the incidence of PMFL $(71 \%)$ and both MFLs was seen in $47 \%$ cases. The incidence of PMFL was higher than incidence of aMFL in most MR images studies. In anatomical studies in the literature, incidences range from 35\% to $76 \%$ for the presence of at least one MFL [12].

In accordance with other authors, we found MFLs in 70 coronal MR images (77.8\%), only aMFL was observed in $20.3 \%$ of cases, PMFL in $37.14 \%$, both ligaments were present in $34.2 \%$ while in $20.3 \%$ MFLs was absent. The results presented in this paper are totally in agreement to the findings of the most studies. However, the authors showed incidence of MFL independent of IN morphology. In our MR images study, we created two IN groups, according to NWI and NSI criteria, $\mathrm{U}$ - and A-shaped notch group. In group with U-shaped IN, we observed at least one MFL with incidence of $84 \%$, while in A-shaped IN group incidence was $70.4 \%$. Occurrence of MFLs obtained in our study is lower in narrowed notch compared with normal sized notch. This probably demonstrates that anatomical changes in this region could have an influence on development and anatomical characteristic of these ligaments. Because of the limitation of the resolution of MR imaging, 15 of the 44 aMFLs, and 2 of the 50 pMFLs did not present details at MR imaging. Similar misreading was noted in literature data [7]. The report of Cho et al. [7] showed that the average running angle was $35^{\circ}$ in the type I, $30^{\circ}$ in the type II and $27^{\circ}$ in the type III. In our study, the average running angle was a little higher in the normal sized IN. The lower value of the running angle in A-shaped IN may be explained by descending medial IN wall, combined with lower ligaments attachment [25]. Statistically significant difference was found in the value of the running angle of PMFL type I, between $\mathrm{U}$ - and A-shaped IN. The running angle modification may be associated with narrowing of the IN, so further studies are needed to investigate this correlation.

\section{CONCLUSIONS}

After conducting the research, we found significant correlation between the IN morphometry and presence or absence of the MFLs as well as the running angle value. The data obtained in our study may be useful in medical clinical practice (reconstructive surgery), interpretation of knee MR images or genetic 
research. Our results suggest that it is possible to determine which patient have narrower IN based on the running angle value. Further study is needed to determine if a correlation exists between morphometry of IN and the value of the running angle.

Funding: This paper was supported by project grants JP 04/18.

\section{Conflict of interest: None declared}

\section{REFERENCES}

1. Amis AA. The biomechanics of ligaments. In: Jenkins DHR (ed.). Ligament injuries and their treatment. Chapman and Hall, London 1985: 3-28.

2. Amis AA, Gupte CM, Bull AMJ, et al. Anatomy of the posterior cruciate ligament and the meniscofemoral ligaments. Knee Surg Sports Traumatol Arthrosc. 2006; 14(3): 257-263, doi: 10.1007/s00167-005-0686-x, indexed in Pubmed: 16228178.

3. Al-Saeed O, Brown M, Athyal R, et al. Association of femoral intercondylar notch morphology, width index and the risk of anterior cruciate ligament injury. Knee Surg Sports Traumatol Arthrosc. 2013; 21(3): 678-682, doi: 10.1007/ s00167-012-2038-y, indexed in Pubmed: 22552619.

4. Anderson AF, Lipscomb AB, Liudahl KJ, et al. Analysis of the intercondylar notch by computed tomography. Am J Sports Med. 1987; 15(6): 547-552, doi: 10.1177/036354658701500605, indexed in Pubmed: 3425782.

5. Balgovind SR, Raunak B, Anusree A. Intercondylar notch morphometrics in Indian population: an anthropometric study with magnetic resonance imaging analysis. J Clin Orthop Trauma. 2019; 10(4): 702-705, doi: 10.1016/j. jcot.2018.07.001, indexed in Pubmed: 31316241.

6. Bintoudi A, Natsis K, Tsitouridis I. Anterior and posterior meniscofemoral ligaments: MRI evaluation. Anat Res Int. 2012; 2012: 839724, doi: 10.1155/2012/839724, indexed in Pubmed: 23019526.

7. Cho JM, Suh JS, Na JB, et al. Variations in meniscofemoral ligaments at anatomical study and MR imaging. Skeletal Radiol. 1999; 28(4): 189-195, doi: 10.1007/ s002560050499, indexed in Pubmed: 10384988.

8. Frank JM, Moatshe G, Brady AW, et al. Lateral meniscus posterior root and meniscofemoral ligaments as stabilizing structures in the ACL-deficient knee: a biomechanical study. Orthop J Sports Med. 2017; 5(6): 2325967117695756, doi: 10.1177/2325967117695756, indexed in Pubmed: 28660229.

9. Moran CJ, Poynton AR, Moran R, et al. Analysis of meniscofemoral ligament tension during knee motion. Arthroscopy. 2006; 22(4): 362-366, doi: 10.1016/j.arthro.2005.09.023, indexed in Pubmed: 16581447.

10. Gupte CM, Smith A, McDermott ID, et al. Meniscofemoral ligaments revisited. Anatomical study, age correlation and clinical implications. J Bone Joint Surg Br. 2002; 84(6): 846-851, doi: 10.1302/0301-620x.84b6.13110, indexed in Pubmed: 12211675.

11. Gupte CM, Bull AMj, Thomas RD, et al. A review of the function and biomechanics of the meniscofemoral liga- ments. Arthroscopy. 2003; 19(2): 161-171, doi: 10.1053/ jars.2003.50011, indexed in Pubmed: 12579149.

12. Heller $L$, Langman J. The menisco-femoral ligaments of the human knee. J Bone Joint Surg Br. 1964; 46: 307-313, doi: 10.1302/0301-620X.46B2.307, indexed in Pubmed: 14167639.

13. Herzog RJ, Silliman JF, Hutton K, et al. Measurements of the intercondylar notch by plain film radiography and magnetic resonance imaging. Am J Sports Med. 1994; 22(2): 204-210, doi: 10.1177/036354659402200209, indexed in Pubmed: 8198188.

14. Hutchinson MR, Ireland ML. Knee injuries in female athletes. Sports Med. 1995; 19(4): 288-302, doi: 10.2165/00007256199519040-00006, indexed in Pubmed: 7604201.

15. Kaplan EB. The lateral meniscofemoral ligament of the knee joint. Bull Hosp Joint Dis. 1956; 17(2): 176-182, indexed in Pubmed: 13413392.

16. Kusayama T, Harner CD, Carlin GJ, et al. Anatomical and biomechanical characteristics of human meniscofemoral ligaments. Knee Surg Sports Traumatol Arthrosc. 1994; 2(4): 234-237, doi: 10.1007/BF01845594, indexed in Pubmed: 8536047.

17. Ohori T, Mae T, Shino K, et al. Complementary function of the meniscofemoral ligament and lateral meniscus posterior root to stabilize the lateral meniscus posterior horn: a biomechanical study in a porcine knee model. Orthop J Sports Med. 2019; 7(1): 2325967118821605, doi: 10.1177/2325967118821605, indexed in Pubmed: 30719478.

18. Röhrich S, Kainberger F, Hirtler L. Evaluation of age-dependent morphometrics of the meniscofemoral ligaments in reference to the posterior cruciate ligament in routine MRI. Eur Radiol. 2018; 28(6): 2369-2379, doi: 10.1007/ s00330-017-5128-x, indexed in Pubmed: 29322332.

19. Shelbourne KD, Davis TJ, Klootwyk TE. The relationship between intercondylar notch width of the femur and the incidence of anterior cruciate ligament tears. A prospective study. Am J Sports Med. 1998; 26(3): 402-408, doi: 10.1177/ 03635465980260031001 , indexed in Pubmed: 9617403.

20. Stijak L, Malis M, Maksimović $R$, et al. [The influence of the morphometric parameters of the intercondylar notch on rupture of the anterior cruciate ligament]. Vojnosanit Pregl. 2012; 69(7): 576-580, doi: 10.2298/vsp101224012s, indexed in Pubmed: 22838168.

21. Tanzer M, Lenczner $E$. The relationship of intercondylar notch size and content to notchplasty requirement in anterior cruciate ligament surgery. Arthroscopy. 1990; 6(2): 89-93, doi: 10.1016/ 0749-8063(90)90004-w, indexed in Pubmed: 2363786.

22. Tillman MD, Smith KR, Bauer JA, et al. Differences in three intercondylar notch geometry indices between males and females: a cadaver study. Knee. 2002; 9(1): 41-46, doi: 10.1016/ s0968-0160(01)00135-1, indexed in Pubmed: 11830380.

23. Tomczyk J, Rachalewski M, Bianek-Bodzak A, et al. Anatomical variations of knee ligaments in magnetic resonance imaging: pictorial essay. Folia Morphol. 2019; 78(3): 467-475, doi: 10.5603/FM.a2019.0004, indexed in Pubmed: 30664231.

24. Yamamoto M, Hirohata K. Anatomical study of the menisco-femoral ligaments of the knee. Kobe J Med Sci. 1991; 37((4-5)), indexed in Pubmed: 1803135.

25. Zhang C, Zhang X, Fang Z, et al. The correlation between common 2D femoral notch parameters and 3D notch volume: a retrospective MRI study. BMC Musculoskelet Disord. 2019; 20(1): 146, doi: 10.1186/s12891-019-2530-3, indexed in Pubmed: 30954066. 